\section{THU0069 RELATIONSHIPS BETWEEN SYNOVIAL FLUID METALLOPROTEINASES AND INTERLEUKIN-1B, AND SERUM CRP IN OSTEOARTHRITIS OF THE KNEE}

M Pianon, L Punzi, A Pozzuoli, F Oliviero, GP Salvati, C Valvason, S Todesco. Division of Rheumatology, Department of Medical and Surgical Sciences, University of Padova, Padova, Italy

\subsection{6/annrheumdis-2001.923}

Background Interleukin (IL)-1 and metalloproteinases (MMP) are thought to play a crucial role in the cartilage degradation of osteoarthritis (OA). Recently it has been proposed that CRP may be useful index of OA activity and severity.

Objectives We studied the relationships between synovial fluid (SF) IL-1b, MMP1 and MMP3, and serum levels of CRP in patients with $\mathrm{OA}$ of the knee (KOA) to investigate the role of CRP in KOA.

Methods In the SF from 25 patients with KOA (Altman criteria, Kellgren and Lawrence score of 2 or greater on X-ray plan films) we determined the levels of IL-1b, MMP-1 and MMP-3 (ELISA), along with WBC number. In the same patients serum concentration of CRP was analysed by ultrasensitive rate nephelometry. No patients were taking NSAIDs or other antirheumatic drugs.

Results In SF, MMP1 was correlated with MMP3 ( $\mathrm{r}=0.61$, $\mathrm{p}$ $=0.0009)$, and MMP3 with IL-1b $(r=0.67, p=0.0002)$. In turn, serum CRP was correlated with MMP3 ( $\mathrm{r}=0.76, \mathrm{p}<$ $0.0001)$ and, at lower level, with MMP1 $(r=0.49, p=0.01)$ and IL- $1 \mathrm{~b}(\mathrm{r}=0.49, \mathrm{p}=0.01)$.

Conclusion The correlations found between serum CRP and SF levels of substances believed to be crucial in OA cartilage degradation, such as MMPs and IL-1, confirm the role of marker of disease severity recently proposed for this acute phase protein.

\section{THU0070 IMMUNOHISTOLOGICAL AND LECTIN HISTOCHEMICAL STUDIES OF STERNOCLAVICULAR AMYLOIDOSIS}

J McClure, N Ghasemi, RW Stoddart, SF McClure. Directorate of Laboratory Medicine, Central Manchester Healthcare Trust, Manchester, UK

10.1136/annrheumdis-2001.924

Background In a prospective necropsy study of the sternoclavicular joints of 31 unselected subjects (15 female, 16 male; mean age 71 years, range 23-92) to ascertain the prevalence of the features of osteoarthrosis, 29 (93.5\%) showed bilateral localised amyloidosis as detected by alkaline Congo red with apple-green dichroism in polarised light. These amyloid deposits were characterised by immunohistological and lectin histochemical techniques.

Objectives

Methods Amyloid deposits were noted in synovial stroma, in the intra-articular fibrocartilaginous disc and in hyaline articular cartilage (usually superficial and middle zones). In synovial stroma only, there were related accumulations of CD68 positive monoand multinucleated cells (37\% of cases). All amyloid deposits stained with anti P-component antibody. None stained with anti AA monoclonal antibody. Ninety per cent of deposits stained with anti Beta 2 microglobulin antibody, $74 \%$ with anti lambda antibody and $61 \%$ with anti kappa antibody (all monoclonal antibodies). Mixed patterns of immunoreactivity were noted.

Results For lectin histochemical studies sections were probed with a panel of 14 biotinylated lectins using techniques standardised in our laboratory. Chondrocytes (including clones) in all layers of articular cartilage and in disc cartilage showed positive cytoplasmic reactions with HHA (Hippeastrum hybrid agglutinin). In addition PSA (Pisum sativum agglutinin) stained particularly the territorial and interterritorial matrices of hyaline articular cartilage in relatively normal and osteoarthrotic cases. E-PHA (Phaseolus vulgaris erythroagglutinin) selectively stained the neomatrix in osteoarthrotic cases.

Conclusion These results showed a high prevalence of localised amyloidosis in the sternoclavicular joint which is not only an age-related phenomenon but is also related to osteoarthrosis, being particularly marked in fibrillated cartilage. A large number of deposits contained Beta2-microglobulin mixed with lambda and kappa light chains and the ubiquitous P-component. Beta2microglobulin amyloidosis is a feature of dialysis amyloid arthropathy. None of the cases in this series were on any form of dialysis and none had impairment of renal function of clinical significance. Light chain amyloidosis usually shows a clonal restriction related to plasma cell dyscrasia/proliferation; the polyclonality and the mixed chemical nature of the amyloidosis reported here is unusual. The lectin profiling showed clear cytoplasmic and matrical differences indicating the presence of nonreducing terminal alpha-mannosyl residues in the former and bi/ tri antennary non-bisected complex $\mathrm{N}$-linked glycans in the latter. Cartilage neomatrix expressed bi/tri antennary bisected complex N-linked glycans. There was no particular relationship between glycan expression and the amyloid deposits. It has been suggested $^{1}$ that the presence of particular glycosaminoglycans in cartilage favours localised amyloidosis either by playing a role in the folding of amyloidogenic proteins in fibril formation or protecting established amyloid deposits from proteolytic degradation. The present results would not support this hypothesis.

\section{REFERENCE}

1 Athanasou NA, West L, Sallie B, Puddle B. Localised amyloid deposition in cartilage is glycosaminoglycans-associated. Histopathology 1995;26:267-72

\section{THU0071 THE ROLE OF TRANSFORMING GROWTH FACTOR B (TGFB) IN BONE METABOLISM IN RHEUMATOID ARTHRITIS}

${ }^{1} \mathrm{~S}$ Drynda, ${ }^{2} \mathrm{G}$ Pap, ${ }^{1} \mathrm{C}$ Kühne, ${ }^{2} \mathrm{~W}$ Neumann, ${ }^{1} \mathrm{~J}$ Kekow. ${ }^{1} \mathrm{Cl}$ linic of Rheumatology; ${ }^{2} \mathrm{Clinic}$ of Orthopedics, Otto-Von-Guericke-University, Magdeburg, Germany

\subsection{6/annrheumdis-2001.925}

Background TGFb can be found in high concentrations in bone matrix. It is produced by osteoclasts and osteoblasts and is considered as a local regulator of bone metabolism. TGFb is an important factor in the coupling of bone resorption and formation. TGFb induces synthesis of matrix proteins and inhibits matrix degradation by downregulation of MMPs and may thereby counteract pro-resorptive cytokines such as IL-1, IL-6 and TNFa. The role of the different isoforms of TGFb is not yet fully understood. In rheumatoid arthritis elevated plasma levels of TGFb1 and TGFb2 have been described.

Objectives It was the aim of this study to determine the role of $\mathrm{TGFb}$ for bone metabolism in RA.

Methods 36 RA patients and 55 patients with osteoarthritis (OA) were included in this study. Total TGFbs in plasma samples and bone extracts were determined after transient acidification applying three isoform specific ELISAs.

Bone mineral density was measured by dexa-scan (Hologic QDR 4500W) at the lumbar spine and femoral neck. Diagnosis of osteoporosis and osteopenia, respectively, was defined according to the WHO osteoporosis criteria from 1994. 
Results Plasma concentrations of TGFb1 were significantly elevated in RA patients compared to OA patients $(7.31 \pm 1.10 \mathrm{ng} /$ $\mathrm{ml}$ vs. $4.11 \pm 0.35 \mathrm{ng} / \mathrm{ml}$, mean \pm SEM, $\mathrm{p}<0.05)$, the other two isoforms did not show differences. Concentrations of TGFbs isolated from bone were strongly associated with each other with a predominance of TGFb1 $>\mathrm{TGFb} 2>\mathrm{TGFb} 3$.

Although the concentrations of TGFb1 in bone were slightly elevated in the RA group compared to the OA group (0.49 \pm 0.07 vs. $0.38 \pm 0.04 \mathrm{ng} / \mathrm{mg}$ bone powder) no significant differences were found. No association was found for TGFb levels in peripheral blood and in the corresponding bone extracts. The highest concentrations of TGFb1 from bone were found in patients with reduced bone mineral density. A strong negative correlation was found between bone TGFb1 levels and T-score values $(\mathrm{r}=-0.479, \mathrm{p}=0.002)$.

Conclusion The finding of elevated TGFb1 concentrations in bone samples from patients with osteoporosis is in contrast to earlier findings, which suggest a protective effect of $\mathrm{TGFb}$ against matrix degradation. In this study the highest concentrations of extractable TGFb1 were found in RA patients with high bone loss. Thus it may be possible that more proinflammatory features of $\mathrm{TGFb}$ are important in bone metabolism, such as the stimulation of IL-6 as described for primary rat osteoblasts. The quantification of total TGFb in the bone matrix may not sufficiently describe the situation in vivo. Post translational processes like activation of the latent $\mathrm{TGFb}$ and its availability determine the biological effects of this regulatory cytokine.

\section{THU0072 DISTRIBUTION OF SELECTIVE MATRIXINS, TIMP-1, CYTOKINES}

J Stovickova, H Hulejova, V Pesakova, M Adam. Institute of Rheumatology, Prague, Czech Republic

\subsection{6/annrheumdis-2001.926}

Background The most abundant joint disease is osteoarthritis. Recently it was classified as cartilagenous, synovial or osseous but the unique forms may be combined. The main feature of the disease is imbalance between synthetic and degradative processes within joint tissues. Executive part in degradative processes play matrix metalloproteinases, so called matrixins. In situ, they are induced by many stimuli, namely by cytokines and by interaction of different adhesive molecules with their ligands. Having been secreted into the extracellular space, they are activated by serine proteinases, or by other matrixins and inhibited by tissue inhibitors of matrix metalloproteinases, TIMPs.

\section{Objectives}

Methods In the sera of OA patients we found matrixins MMP-9, MMP-2 and MMP-3 and TIMP-1 together with cytokines IL-8 and TNF/alpha and adhesive molecules ICAM-1 and VCAM-1. To distinguish between the forms of osteoarthritis, we search for these molecules in all joint tissues that is in cartilage, synovium, granulation tissue, within subchondral bone, synovial fluid and blood serum. The joint tissues and fluids we obtain from surgery during total hip or knee replacement.

Results The joint tissues were derived from 22 patients. We obtained 10 samples of knee joints and 12 samples of hip joints. The patients were 17 female and 6 male, 67,5 \pm 9,6 years old, age ranges $48-86$ years. The metalloproteinase spectrum in the body fluids and extracts of joint tissues were evaluated by zymography. The concentration of metalloproteinases and TIMP1 were assessed by ELISA (Biotrak, Amersham Pharmacia
Biotech), the concentration of adhesive immunoglobulins by Bender ELISA (MedSystems Diagnostics $\mathrm{GmbH}$ ), and the concentration of IL-8 and TNF/alpha were determined on Immulite (DPC, USA).

We found that the most abundant matrixine within cartilage and synovium is either gelatinase A (MMP-2) or stromelysine-1 (MMP-3). On the other hand, within granulation tissue, blood sera and synovial fluid, the prevailing matrixine is gelatinase $\mathrm{B}$ or stromelysine-1. In cartilage, synovial fluid and blood serum TIMP-1 is abundant over sum of matrixins, whereas in synovium and granulation tissue the sum of matrixins is abundant over TIMP-1. There is about 2 to 10 times greater concentration of IL- 8 in the cartilage than in other joint compartments.

We found significant correlation between levels of sICAM-1 and sVCAM-1, between TNF/alpha and MMP-3, TIMP-1, sICAM-1, sVCAM-1, and IL-8, and between IL-8 and sICAM-1 in all solid joint tissues. In the synovial fluid and blood serum the correlation was less pronounced.

Conclusion There is a great degradative potential within all compartments of osteoarthritic joint due to presence of matrixins, namely that of stromelysin-1. Within cartilage and synovial fluid the proteolytic potential is equilibrated by TIMP-1 whereas within synovium and granulation tissue is not. Synovium and granulation tissue are highly probably the propellers of degradative process in osteoarthritis.

Rheumatoid Arthritis - Aetiology and pathogenesis/Animal models

\section{AB0022 LACTOFERRIN - A POTENT ANTI-INFLAMMATORY AGENT FOR TREATMENT OF ADJUVANT ARTHRITIS}

AV Danilov, NI Korshunov, TG Danilova, VP Mikhailov, LA Tsyganova. Internal Diseases, Yaroslavl State Medical Academy, Yaroslavl, Russia

10.1136/annrheumdis-2001.927

Background Lactoferrin is a multifunctional immunoregulatory protein, stored in specific granules of neutrophil granulocytes, from which it is released following cell activation. While the biochemical characterisation of lactoferrin as an iron-binding protein has been well described, its physiological role in inflammation remains undefined. Although lactoferrin was proven to exert certain antiinflammatory effects, there is a paucity of data concerning the possibility of administrating lactoferrin as an anti-inflammatory agent in rheumatoid arthritis.

Objectives To evaluate the anti-inflammatory properties of lactoferrin in treatment of adjuvant arthritis ? an adequate model of rheumatoid arthritis.

Methods Adjuvant arthritis was induced in Lewis rats by subplantar inoculation of a complete Freund?s adjuvant. Human milk-derived lactoferrin was administered intravenously in the acute phase (8th day after adjuvant inoculation), and in the chronic phase of adjuvant arthritis (29-31 days).

Results The development of adjuvant arthritis in rats was characterised by hind paws swelling and hyperthermia, weight loss, increased white blood cell count and erythrocyte sedimentation rate, an increased level of serum elastase. In approximately 50\% of rats a generalisation of inflammation with involvement of intact joints was observed. Treatment with low doses of lactoferrin $(3-18 \mathrm{mg} / \mathrm{kg})$ in the acute phase of adjuvant arthritis did not change the course of the disease, while higher doses of lactoferrin $(30-60 \mathrm{mg} / \mathrm{kg}$ ) caused a certain suppression of the inflammatory process, although it was not stable and lasted for only 2 